\title{
Suicidio por decapitación con sierra mecánica
}

\section{Suicide by decapitation using a tapesaw}

\section{Resumen}

Se expone un caso de decapitación suicida realizada con una sierra de cinta para cortar metales. Precisó una laboriosa preparación previa en el taller de metalistería donde se ubicaba la máquina. El corte se realizó de delante a atrás con dirección oblicua, seccionando la escama del occipital en gran parte.

Palabras clave: Decapitación suicida. Sierra de cinta. Marcas de corte. Hueso humano.

\section{Abstract}

A suicide decapitation case is presented that was committed using a tapesaw to cut metals. It required a previous laborious preparation at the metalwork place where the machine was located. Cut was made from the front to back with an oblique direction which caused the cut of a large part of the occipital squama.

Key words: Suicide decapitation. Tapesaw. Cut marks. Human bone.

\section{Antecedentes del caso}

Se trata de un varón de 22 años, sin antecedentes psiquiátricos constatados, aunque según parece con algunos problemas personales. En el lugar del levantamiento aparece una nota de suicidio escrita en el reverso de un sobre que se encontró a los pies del cadáver. El cadáver se halla en una nave-taller de metalistería, sobre la bancada de una máquina herramienta eléctrica usada para el corte de metales.

El individuo prepara la escena y, para ello, acerca un remolque a la bancada metálica de la sierra eléctrica de cinta, dispone una viga en doble T que hace de contrapeso para vencer el muelle recuperador del área de corte y coloca una brida de plástico para mantener la presión sobre la tecla del interruptor de contacto (Figura 1).

El cadáver se encuentra tendido en decúbito supino, con las piernas abiertas, la izquierda sobre el remolque, y los brazos colgando a ambos lados del cuerpo (Figura 2).
La cabeza está seccionada en su práctica totalidad, pues sólo se mantiene un pequeño colgajo de piel en la porción posterior izquierda. En la bandeja de la máquina se encuentra la mayor parte de la cabeza sobre un charco de sangre.

En el suelo se aprecian manchas de proyección oblicuas de sangre, que siguen una línea prácticamente recta como resultado de la sección de los vasos a gran velocidad (Figura 3 ).

El corte sigue una línea oblicua de delante a atrás que interesa la parte superior del cuello y la inferior del cráneo.

\section{Hallazgos de autopsia}

\section{Examen externo}

El cadáver presenta una sección prácticamente completa de la cabeza, apreciándose una única línea de decapitación que comienza inmediatamente por

\section{B. Rodríguez Hidalgo \\ I. Martínez Téllez ${ }^{1}$ \\ MC. Botella López ${ }^{2}$}

${ }^{1}$ Médico Forense del Instituto de Medicina Legal de Granada. Servicio de Clínica Forense.

${ }^{2}$ Antropólogo forense. Laboratorio de Antropología Física, Facultad de Medicina, Universidad de Granada.

Correspondencia:

Dra. Beatriz Rguez. Hidalgo E-mail:

beatriz.rodríguez.hidalgo@ juntadeandalucia.es

Fecha de recepción: 14.OCT.2010

Fecha de aceptación: 19.OCT.2010 

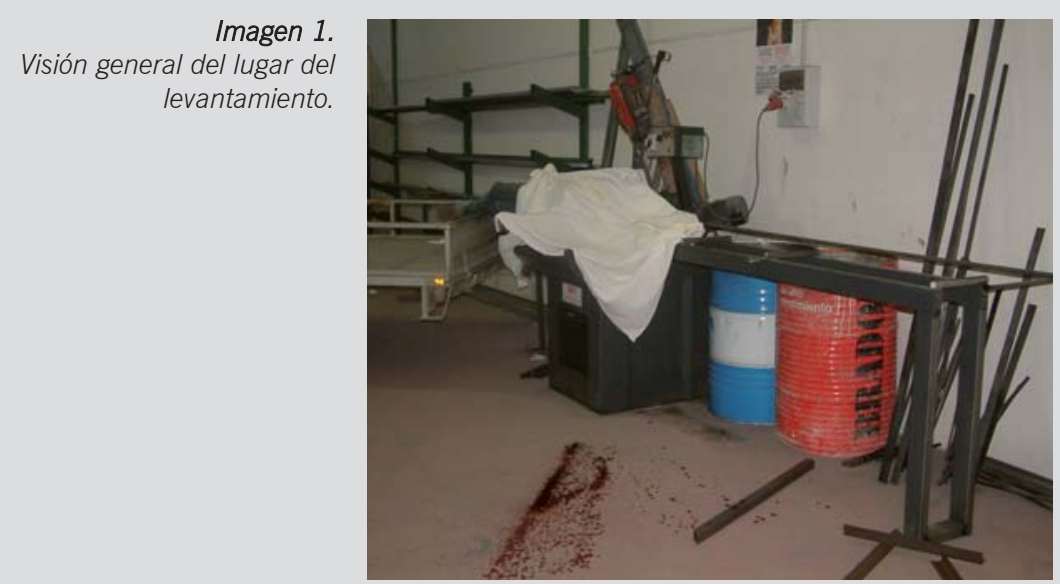

Imagen 2.

Posición del cadáver en la

bancada y detalle del mecanismo suicida utilizado.

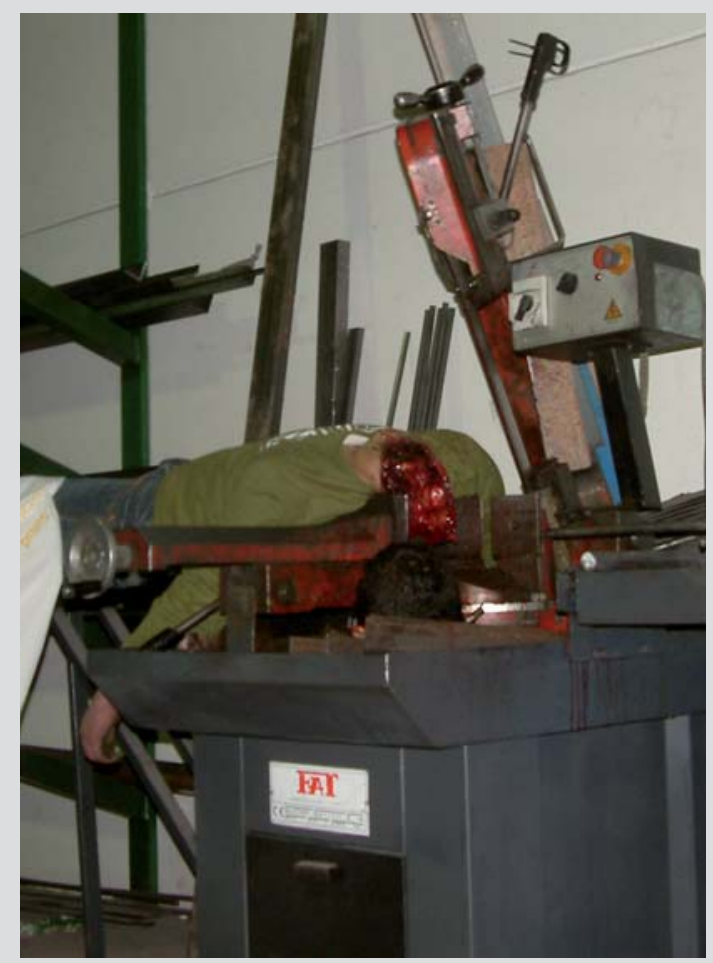

Imagen 3.

Mancha de sangre en proyección oblicua.

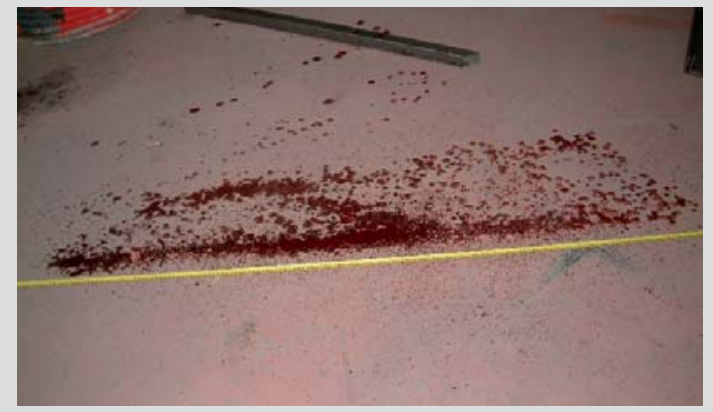

encima del cartílago tiroides y continúa en una línea ininterrumpida ascendente hasta la parte media de la escama del occipital. Está cortado el vértice del lóbulo del pabellón auricular derecho.

Sólo conserva como unión de los dos segmentos un pequeño trozo de cuero cabelludo en la parte izquierda de la región occipital.

De la observación del corte (Figura 4), destaca su regularidad y que afecta a orofaringe a la altura de la base de la lengua, médula espinal con sección completa, cerebelo en su mitad y ambos lóbulos occipitales, con mayor afectación del derecho.

Los bordes son suaves, no invertidos y con ribete contusivo uniforme excepto en la parte posterior izquierda donde se hace irregular.

No se encuentra ningún otro signo de violencia. El resto de órganos y estructuras de tórax y abdomen no muestran lesiones traumáticas, presentando un patrón macroscópico dentro de la normalidad para la edad del fallecido. El cuerpo está exangüe.

En el análisis químico-toxicológico se detecta la presencia de cocaína y sus metabolitos metilecgonina y benzoilecgonina, así como etilbenzoilecgonina (consumo simultáneo de cocaína y alcohol etílico). Las concentraciones halladas se encontraban dentro de los rasgos de consumo semireciente y antiguo.

\section{El corte}

Se limpia la porción de cráneo que comprende parte de la escama occipital y temporal desde la mastoides derecha, y visto con luz incidente tangencial se observan las marcas de corte.

Se aprecian estrías paralelas en toda la superficie con manifiestas irregularidades en la anchura y en la profundidad, lo cual indica que la sierra eléctrica estaba muy usada y ya tenía un avanzado desgaste de los dientes. La velocidad de giro de la cinta debió ser elevada porque las líneas de los cortes son limpias y no presentan arrollamientos del hueso.

Posiblemente algunos dientes estaban o muy desgastados o faltaban completamente, ya que se aprecian huecos en el hueso que corresponden al espacio entre uno o dos dientes (Figura 5).

\section{Discusión}

La muerte por decapitación, en su origen, puede corresponder tanto a un suicidio como a un homicidio, 


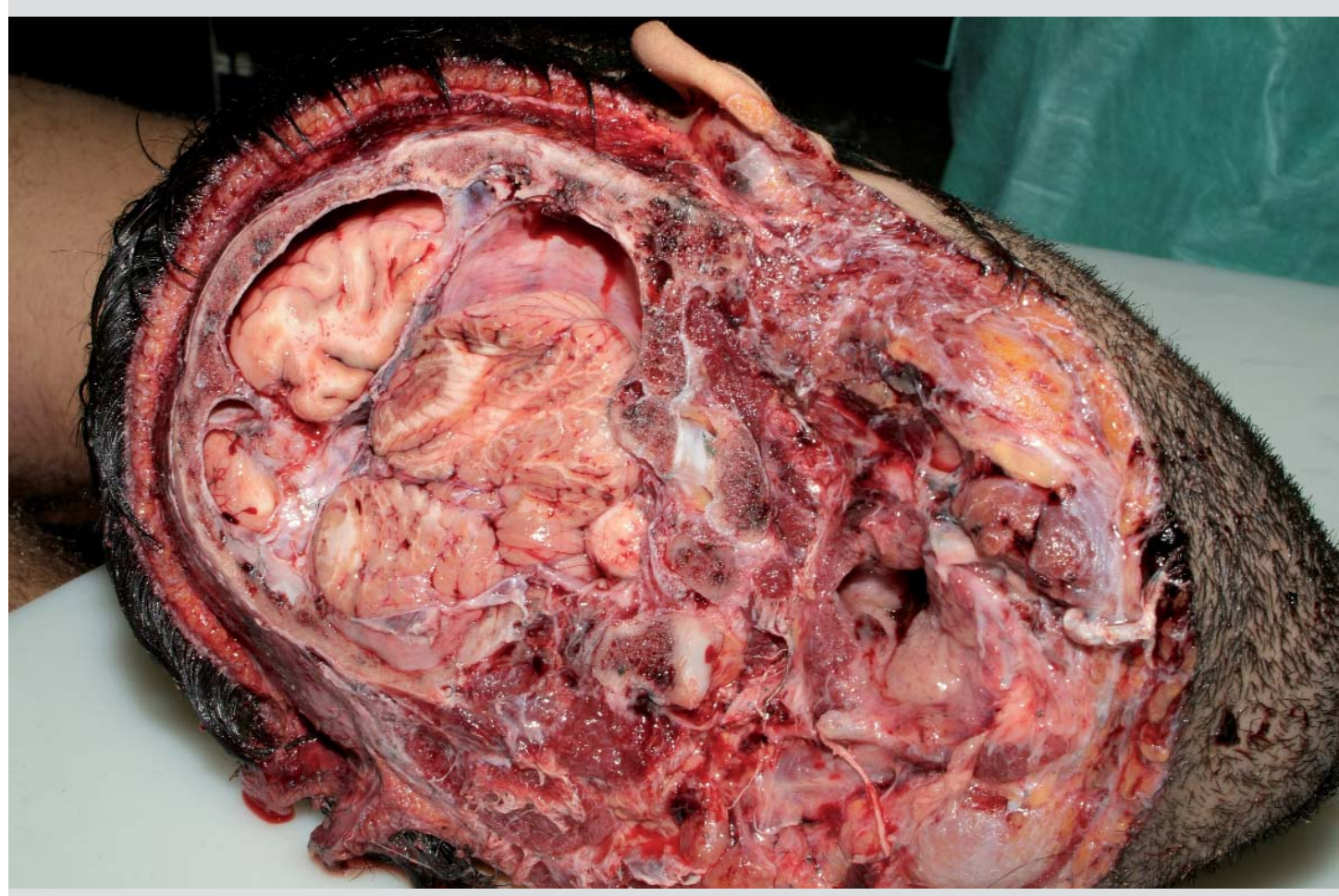

Imagen 4.

Sección del hueso visto en la autopsia.

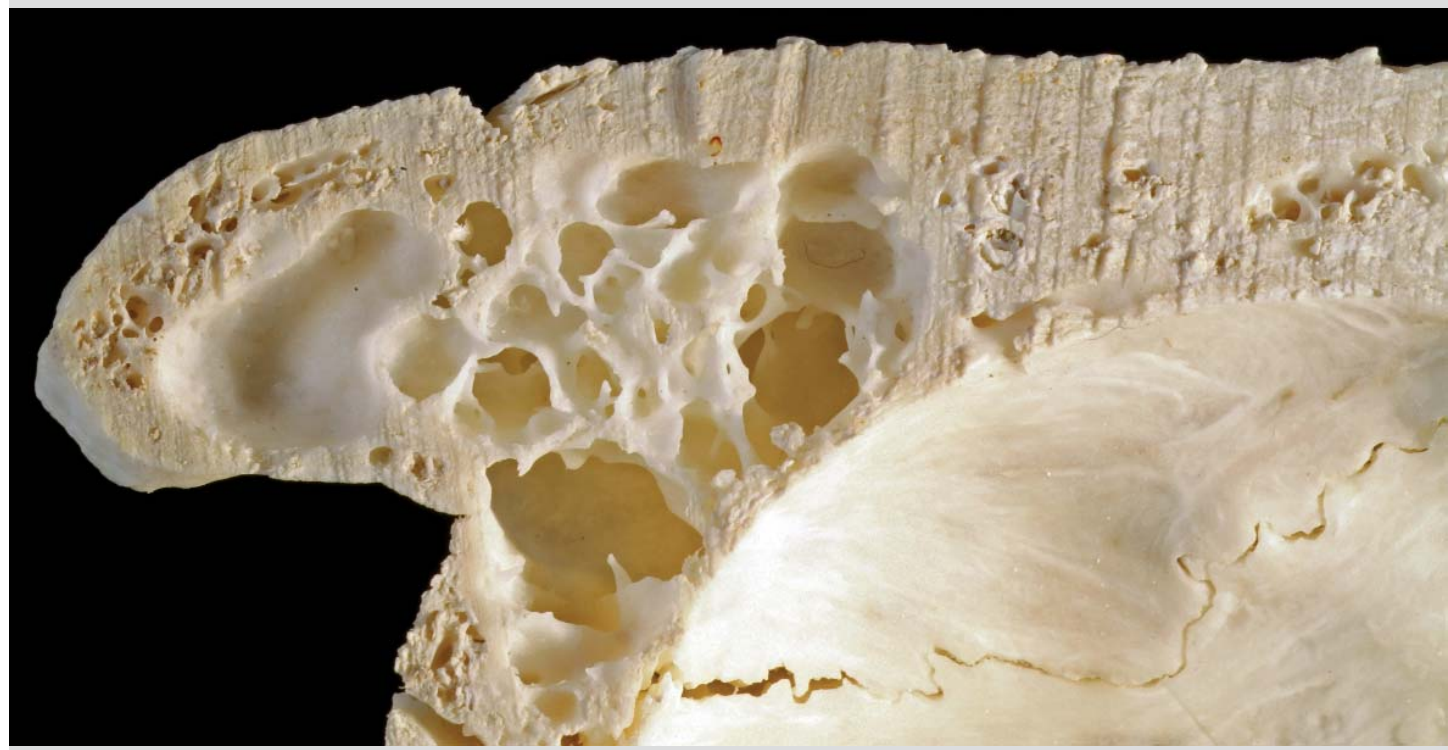

Imagen 5.

Marcas del corte del hueso visto con luz rasante.

sin estar excluida la etiología accidental. No obstante, las heridas suicidas no pueden tener una gran profundidad, por la dificultad de alcanzar esta región anatómica la propia víctima con la necesaria energía ${ }^{1}$. La importancia de este caso radica en la escasez de las decapitaciones de naturaleza suicida en la literatura médico-legal y la rareza del hallazgo publicado. En publicaciones anteriores se han descrito algunos casos de decapitación suicida, pero en el contexto de otros mecanismos de igual etiología como la ahorcadura. Asimismo existen otros de decapitaciones autolíticas en vía férrea, aunque en 
nuestro medio la etiología más frecuente es la de carácter accidental (accidentes de tráfico y accidentes laborales).

Es más común, sin embargo, la etiología homicida, por lo que es necesario en este tipo de muertes plantearse el diagnóstico diferencial entre un verdadero suicidio o si se trata de un homicidio que ha intentado ser disimulado como tal.

Dado lo inusual de este caso, se considera de especial interés realizar un estudio detallado en el lugar de los hechos (de la preparación y de los mecanismos utilizados en la manipulación de la maquinaria, así como de los restos biológicos dejados) previo al estudio necrópsico.

Las células del tejido óseo se encuentran en constante e intensa actividad, y es notable su capacidad de reparación de las zonas dañadas. Por eso, las marcas de manipulación sólo podrán observarse cuando se produjeron en un estadio perimortem, cuando el hueso no tuvo tiempo de reaccionar ante la agresión. Las señales que se observan son, por lo tanto, las de la alteración de la integridad en ese tejido, cuando no ha actuado la capacidad regeneradora de los osteocitos. Se compone de elementos orgánicos e inorgánicos, y esto hace que posea rasgos específicos, interesando en este caso destacar la relativa estabilidad y capacidad de mantener las estructuras. Tienen, asimismo, capacidad para conservar huellas de las manipulaciones que hayan quedado señaladas, las cuales perdurarán en el tiempo después de que el sujeto haya muerto. Cuando las células del tejido óseo tienen tiempo de restaurar las estructuras, las marcas desaparecerán o al menos se irán atenuando, a veces con notables cambios en su apariencia. Pero si se producen en un estadio perimortem, no hay evidencias de regeneración ósea. El estado físico del hueso cambia de manera considerable si está fresco o desvitalizado (sin su

\section{Bibliografía}

1. Gisbert Calabuig JA. Lesiones por arma blanca. En: Gisbert Calabuig JA, Villanueva Cañadas E. Medicina legal y toxicología. $6^{a}$ ed. Barcelona: Editorial Masson, 2004;383-93.

2. Botella M, Alemán I, García C. Marcas de huesos humanos. En: Campillo D. Introducción a la paleo- parte orgánica), por lo que se puede diferenciar, según el estado de las huellas, si existió manipulación².

Con respecto al corte del hueso, los indicios que marcan la diferencia respecto a la superficie pulida de un hueso por abrasión son básicamente la convexidad, las incisiones que se aprecian en el lugar de comienzo y las rebabas del final.

Los utensilios dentados necesitan de un movimiento de vaivén para cortar. En cada uno de ellos, la sierra se hunde un poco más en el hueso y deja un pequeño escalón. Por eso se ve estriada la sección de un hueso cortado con sierra. Esas estrías serán tanto más anchas cuanto mayor sea el tamaño de los dientes. Su profundidad y número dependerá de lo aguzado que esté y de la fuerza que se haya empleado en la maniobra.

Siempre son paralelas entre si y su regularidad está en relación con la fuerza utilizada. Si ésta se mantiene aproximadamente igual, serán sensiblemente iguales en anchura, pero si es variable, también lo serán las huellas ${ }^{3,4}$.

\section{Conclusiones}

La decapitación suicida es un mecanismo de muerte poco frecuente en nuestro medio, por lo que es necesario establecer diagnóstico diferencial en relación a las distintas etiologías médico-legales, comenzando dicho diagnóstico en la inspección ocular del levantamiento de cadáver. Desde el punto de vista antropológico se puede llevar a cabo una correlación entre las marcas dejadas en el hueso y el utensilio utilizado. En este caso, las marcas dejadas en el hueso fresco son características del utensilio sierra y específicas de la máquina utilizada y encontrada en el lugar del levantamiento. patología. $1^{\text {a }}$ ed. Barcelona: Editorial Bellaterra, 2001;408-20.

3. Botella M, Alemán I, Jiménez S. Los huesos humanos. Manipulación y alteraciones. $1^{\text {a }}$ ed. Barcelona: Editorial Bellaterra, 2000.

4. Botella M, Alemán I. Las huellas del canibalismo. Arch Esp Morfol 1998;3:75-86. 